\title{
Random walks with absolute negative mobility
}

\author{
B. Cleuren and C. Van den Broeck \\ Limburgs Universitair Centrum, B-3590 Diepenbeek, Belgium \\ (Received 5 October 2001; published 8 February 2002)
}

\begin{abstract}
We introduce simple non-Markovian modifications to the standard random walk resulting in absolute negative mobility, i.e., the response to an external force is always opposite to the direction of the force.

DOI: 10.1103/PhysRevE.65.030101

PACS number(s): 05.40.-a, 02.50.-r
\end{abstract}

\section{INTRODUCTION}

The random walker is a basic paradigm in science that has been applied to a wide range of problems in many different fields $[1,2]$. In statistical mechanics and the theory of stochastic processes, it has been studied in great detail and provides a technically simple and conceptually transparent discretized version of the Wiener process. When refering to the erratic motion of thermally agitated particles, the latter is also known as Brownian motion. The rectification of Brownian motion is forbidden by the second law of thermodynamics in a system at equilibrium. However, work can be extracted from this thermal motion when operating under nonequilibrium conditions, as demonstrated explicitly in the various models for so-called Brownian motors [3]. Another limitation of equilibrium is related to the linear response properties. Motion on the average in a direction against a small external force is impossible. In particular, the mobility coefficient $\mu$ relating the drift velocity of a Brownian particle (diffusion coefficient $D$ ) in linear response to an external force is positive as made explicit in the Einstein relation $\mu=D / k_{B} T$. In nonequilibrium, however, there is no fundamental principle that forbids absolute negative mobility. In fact, electrical networks with negative resistance have been known for a long time [4]. More recently, various specific examples of Brownian motors have been introduced $[5,6]$ that display negative linear mobility. In this paper, we move away from a detailed physical model to show how very simple non-Markovian modifications of the basic random walk can result in absolute negative mobility, thereby putting this phenomenon in a much broader context.

\section{RANDOM WALK MODEL}

We start from the usual discrete time random walk of a particle taking steps of size one to the left or to the right with probabilities $p$ and $q=1-p$, respectively. On average, the particle will acquire a drift velocity equal to $2 p-1$. A well known non-Markovian modification is the so-called random walk with persistence, in which the step directions taken by the walker are correlated with previous ones. In the random walk modification presented here, it is the stepsizes that are correlated, rather than their direction. The rule is the following. A particle performs the above described biased random walk. We introduce a new parameter, denoted by $N-1$, which gives the maximal allowed number of consecutive steps in the same direction. Hence, whenever the particle hops in the same direction for $N$ successive time steps, this excursion is cancelled, i.e., the particle is transferred back to the original position of $N$ steps ago, and its memory is cleared. Our main purpose now is to study how this limitation of large excursions impacts the resulting drift velocity. To do so, we note that the state of the particle is fully described by the knowledge of its position $x$, together with the length $l$ of the last sequence of successive steps in the same direction $(l \in\{ \pm 1, \ldots, \pm N\})$. The probability $P(x, l, n)$ to find the system in state $(x, l)$ after $n$ steps obeys the following master equation:

$$
\begin{gathered}
P(x, N, n)=p P(x+N-1, N-1, n-1), \\
P(x, j, n)=p P(x-1, j-1, n-1), \\
P(x, 1, n)=p P(x-1, N, n-1)+p \sum_{m=-1}^{-N} P(x-1, m, n-1),
\end{gathered}
$$

with initial conditions $P(x, l, 0)=\frac{1}{2} \delta_{x, 0} \delta_{l, \pm N}$. A similar set of equations applies for the steps to the left (probability $q=1$ $-p$ ). From Eq. (1), one obtains the following exact result for the Fourier transform of the generating function:

$$
\begin{aligned}
F(k, z) & =\sum_{x=-\infty}^{\infty} e^{i k x} \sum_{n=0}^{\infty} z^{n} P(x, n) \\
& =\frac{1}{f(p, k)+f(1-p,-k)-1},
\end{aligned}
$$

with $P(x, n)=\sum_{l= \pm 1}^{ \pm N} P(x, l, n)$ and

$$
f(p, k)=\left(1-z p e^{i k}\right) \frac{1-(z p)^{N}}{1-\left(z p e^{i k}\right)^{N}}
$$

In the long time limit $n \rightarrow \infty$, the distribution becomes Gaussian and is characterized by the following drift velocity $v$ and diffusion coefficient $D$ (see Appendix):

$$
\begin{aligned}
v & =\lim _{n \rightarrow \infty} \frac{\langle x(n)\rangle}{n} \\
& =2 p-1+\frac{N(1-p)^{N} p}{1-(1-p)^{N}}-\frac{N(1-p) p^{N}}{1-p^{N}},
\end{aligned}
$$




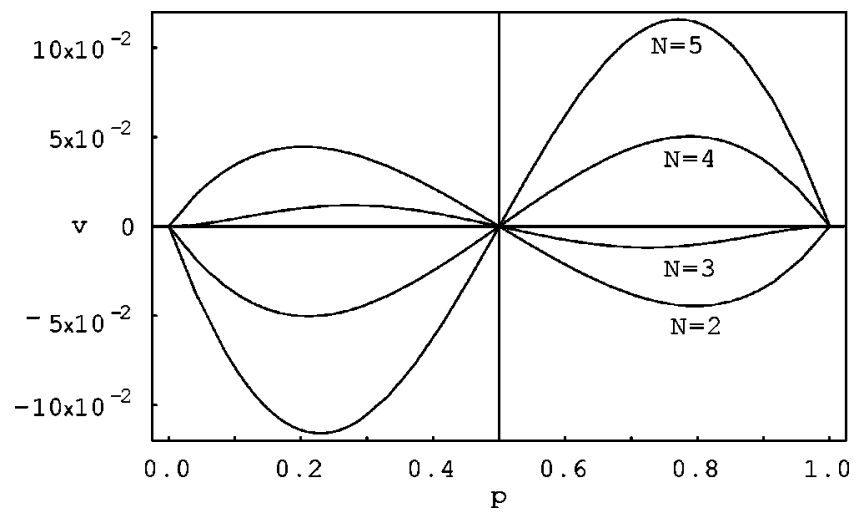

FIG. 1. The average speed $v$ as a function of the bias $p$ for different values of $N$. For $N=2$ and 3 one observes negative mobility.

$$
\begin{aligned}
D= & \lim _{n \rightarrow \infty} \frac{\left\langle x^{2}(n)\right\rangle-\langle x(n)\rangle^{2}}{2 n} \\
= & \frac{N^{3}}{\left(1-q^{N}\right)^{3}\left(1-p^{N}\right)^{3}} \\
& \times\left[\begin{array}{l}
\left\{p^{2 N+1} q^{N}-p^{N} q-2 p^{N}(p-q) q^{N}+p q^{N}\right. \\
\left.-p^{N} q^{2 N+1}\right\}\left\{p^{N} q+p^{N}(p-q) q^{N}-p q^{N}\right\}
\end{array}\right] \\
& +\frac{N^{2}}{2\left(1-q^{N}\right)^{2}\left(1-p^{N}\right)^{2}} \\
& \times\left[\begin{array}{l}
p^{N} q^{N}\left\{p^{N}(2 q-p)+q^{N}(2 p-q)-14 p q\right. \\
\left.+4-2 p^{N} q^{N}\left(p^{2}+q^{2}\right)\right\}+p q\left(p^{2 N}+q^{2 N}\right) \\
+p^{N} q(4 p-3)+p q^{N}(4 q-3)
\end{array}\right] \\
& +\frac{N}{\left(1-p^{N}\right)\left(1-q^{N}\right)}\left[2 p q\left\{p^{N}-2 p^{N} q^{N}+q^{N}\right\}\right]+2 p q .
\end{aligned}
$$

Equation (4) is a central result of this paper. In Fig. 1, the dependence of $v$ on the bias $p$ is plotted for different values of $N$. The key observation is that the particle moves in a direction opposite to the bias for $N=2$ and 3 . The intuitive reason is that the penalization of large excursions is strongest in the direction of the bias.

For the sake of completeness, we also represent the diffusion coefficient as a function of $p$ in Fig. 2, for various values of $N$. Note the development of two symmetric peaks of increasing height as $N$ becomes larger. This increased dispersion is due to the large steps corresponding to cancelled excursions of size $N$. To have enough probability weight, such excursions, however, require a stronger bias when $N$ becomes large.

\section{APPLICATIONS}

We mention three examples that can be mapped on this type of random walk. The first one comes from chemistry. A linear polymer is growing in a mixture of $A$ and $B$ molecules,

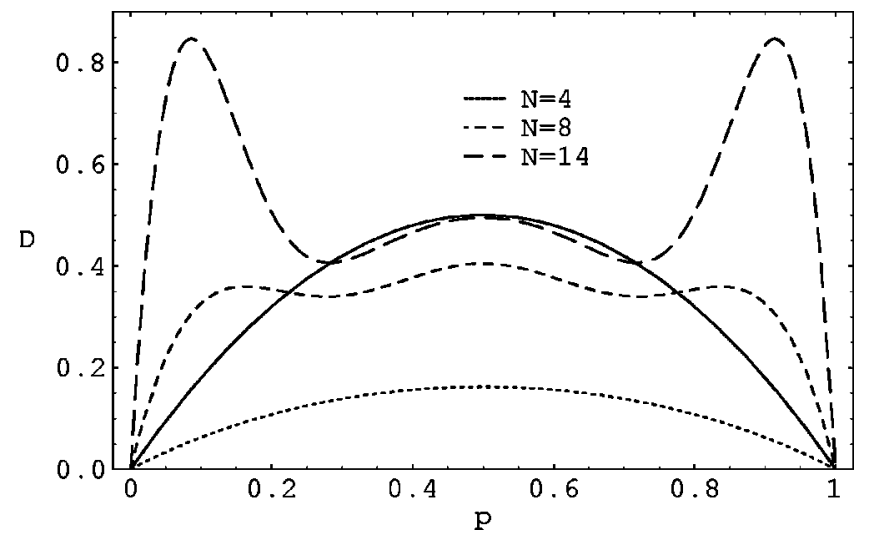

FIG. 2. The diffusion coefficient $D$ as a function of the bias $p$ for different values of $N$. The solid line represents the diffusion coefficient for the normal random walk.

with concentrations $c_{A}$ and $c_{B}$, respectively. They attach to the polymer with a probability proportional to their relative concentration, i.e., the probability that an $A$ particle is chosen reads $p=\left[c_{A} /\left(c_{A}+c_{B}\right)\right]_{\text {mixture }}$. When two identical molecules attach successively, they dimerize and disconnect from the polymer. If we represent the succession of $A$ and $B$ molecules along the polymer as steps to right and left, respectively, of a random walk, we have a realization of our model with $N=2$. The long time relative concentrations of $A$ versus $B$ in the polymer is given by the drift velocity $v$ $=2\left[c_{A} /\left(c_{A}+c_{B}\right)\right]_{\text {polymer }}-1$. Hence negative mobility implies that the polymer will be rich in the species that is poor in the mixture.

Second, we turn to an example from physics. A charged particle is performing a cycloid motion in the $(x, y)$ plane orthogonal to a magnetic field along the $z$ direction, with an electric field parallel to the $y$ axis [7]. At each multiple of half the cyclotron period, one chooses the sign of magnetic field to be either positive or negative with probabilities $p$ and $q=1-p$, respectively. The sign of the electric field is switched if that of the magnetic field has not changed, otherwise it is taken to be positive. Clearly, when the magnetic field is switched to a positive value, the particle will rotate to a new position in the positive $x$ direction. As long as the sign of this field does not change, the particle will rotate back and forth between this new position and its original position. A similar dynamics takes place for negative magnetic field, but taking place now in the negative $x$ direction. The position of the particle at half multiples of the period thus undergoes the above described random walk, again with $N=2$. Negative mobility implies that the drift motion along the $x$ axis will be opposite to what is expected from the dominant direction of the magnetic field.

As a last example, we construct a new paradoxical game [8]. A banker offers an investment with, on average, a positive yearly return. In our random walk model, the probability for winning a unit of capital is $p>1 / 2$. A nervous investor wants more protection against losses. The banker proposes to cancel the losses in $N$ successive years. But he argues that, in all fairness, he then also has to cancel the wins in $N$ successive years. The capital of the investor now undergoes the 
above described random walk with the paradoxical result that the investment will have on average a negative return if $N=2$ and 3 .

\section{COLLECTIVE EFFECTS}

The random walk model introduced above is a prototype. Many variations and modifications can be envisaged. We discuss next, in some detail, possible cooperative versions. We cite two prominent mechanisms that may appear quite naturally when the walkers interact: crowding and greediness. As an example of crowding, consider a set of Brownian particles in a channel with a periodic array of gates. Note that such a setup can be easily realized experimentally using, for example, latex particles suspended in a flow through fabricated micropores in silicon wafers [9]. The gates should be such that a single particle can easily pass through while the passage is hampered when several particles are present on the same side of the gate. Upon application of an external bias, such a crowding will appear more frequently on the side of the gates that is upstream of the force, with negative mobility as a possible outcome. We stress that this intuitive argument can be misleading. Indeed, as already mentioned in the Introduction, negative mobility is ruled out near equilibrium by the fluctuation dissipation theorem. One thus either has to work in the regime of nonlinear response, or linear response around a nonequilibrium state. An explicit example of the latter case is given by the model introduced in Ref. [6], where the narrow gate (represented by a barrier) is also cooled. A biochemical alternative corresponds to a gate that operates unbiased active transport, which is deactivated when more then one particle attaches to the same site.

The second mechanism, greediness, is more intricate and possibly more surprising: it involves informed walkers that modify their jump statistics in a greedy way in response to the motion of the other walkers. A deterministic analog is the so-called Braess paradox [10], in which it is found that the addition of a new route to go from one point to another actually slows down the traffic in that direction. The opening of the new route makes the original flow pattern unstable against greedy defectors, who, by choosing a trajectory that is faster for them with respect to the original flow pattern, produce a cascade of modifications leading to a stable but suboptimal equilbrium. The paradox is also well known in macroeconomics where these type of suboptimal states are referred to as Nash equilibria. In short, just like in the traffic problem, the pursuance of individual interests can be the demise of a collective goal. In the context of random walkers, one can imagine negative mobility to appear when, as a result of the installation of a bias, walkers greedily choose routes that appear, with respect to the existing flow patterns, favourable to themselves, but effectively result in an overall delay of the motion in the direction of the bias.

\section{DISCUSSION}

We close with a discussion of the distinctive features of walks with negative mobility and a broad overview of related results from the literature. First, such walkers are reservoirs of energy that become available upon request. Rather than dissipating energy supplied by an external force, the walker, which moves in a direction opposite to the force, actually performs work. Usual Brownian motors also have this capability, but in the present case energy is only released when loading takes place. Concomitantly, the direction of the loading is irrelevant. Second, whereas usual walkers get trapped in the minima of an external potential, the ones with negative mobility move towards the maxima. The roles of stability and instability are thus interchanged. This property might conceivably play a role in triggering a chemical reaction with the walker as catalyst. Third, other related results from the literature include linear response around nonequilibrium steady states, reconsidered in the light of anomalous response properties in Ref. [11], and negative mobility in networks with dead ends [12]. Finally, negative mobility can also appear when the force acts, not only on the walkers, but also, directly or indirectly, on the substrate, see, for example, the well known anomalous transport of electrons [13].

\section{ACKNOWLEDGMENTS}

We thank the Program on Inter University Attraction Poles of the Belgian Government and NSF Grant No. PHY9970699 for financial support.

\section{APPENDIX}

In this appendix, we show how to obtain the velocity $v$ and diffusion coefficient $D$ from the Fourier transform $F(k, z)$ of the generating function. Expanding $F(k, z)$ around $k=0$ gives

$$
F(k, z)=\sum_{m=0}^{\infty} \frac{\left\langle x^{m}(z)\right\rangle}{m !}(i k)^{m}
$$

where we define $\left\langle x^{m}(z)\right\rangle \equiv \sum_{n=0}^{\infty}\left\langle x^{m}(n)\right\rangle z^{n}$. The asymptotic behavior of the moments can be obtained by making use of the so-called Tauberian theorems. In the case of a power series, such as $f(z)=\sum_{n=0}^{\infty} f_{n} z^{n}$, they state that the asymptotic dependence of $f_{n}$ for $n \rightarrow \infty$ is closely related to the singular behavior of $f(z)$. A useful theorem is the following [2]: Let $f(z)=\sum_{n=0}^{\infty} f_{n} z^{n}$, with $f_{n}$ a strictly positive and monotonic function of $n$. If $f(z)$ is singular in the limit $z$ $\rightarrow 1$,

$$
f(z) \sim \frac{L\left(\frac{1}{1-z}\right)}{(1-z)^{\alpha}},
$$

with $L(x)$ a slowly varying function and $x^{\alpha} L(x)$ a positive monotonically increasing function of $x$ for large $x$. Then, in the limit $n \rightarrow \infty$,

$$
f_{n} \sim \frac{\alpha n^{\alpha-1} L(n)+n^{\alpha} L^{\prime}(n)}{\Gamma(1+\alpha)} .
$$

For the first moment $\langle x(n)\rangle$ one finds from Eq. (3) and (A1), 


$$
\langle x(z)\rangle \sim \frac{v}{(1-z)^{2}},
$$

where $v$ is the speed given by Eq. (4). Applying the above theorem for $\alpha=2$ and $L(x)$ a constant equal to $v$, we conclude that $\langle x(n)\rangle \sim n v$ for large $n$. The diffusion coefficient $D$ can be obtained in a similar way, by considering $y(n)$ $\equiv x(n)-\langle x(n)\rangle$ with $D=\lim _{n \rightarrow \infty}\left\langle y^{2}(n)\right\rangle / 2 n$. Note that this requires the calculation to the next order in the asymptotic behavior of $\langle x(n)\rangle$, namely,

$$
\begin{gathered}
\langle x(z)\rangle-\frac{v}{(1-z)^{2}} \sim \frac{C-v}{1-z}, \\
C=N\left[\frac{1-q^{N}(1+N p)}{\left(1-q^{N}\right)^{2}}-\frac{1-p^{N}(1+N q)}{\left(1-p^{N}\right)^{2}}\right] .
\end{gathered}
$$

[1] H. C. Berg, Random Walks in Biology (Princeton University Press, Princeton, 1983); E. W. Montroll and M. F. Shlesinger, The Wonderful World of Random Walks in Nonequilibrium Phenomena II. From Statistics to Hydrodynamics, edited by J. L. Lebowitz and E. W. Montroll (North-Holland, Amsterdam, 1984); R. M. Mazo (unpublished).

[2] G. H. Weiss, Aspects and Applications of the Random Walk (North-Holland, Amsterdam, 1994).

[3] F. Jülicher and J. Prost, Prog. Theor. Phys. Suppl. 130, 9 (1998); C. Van den Broeck, P. Reimann, R. Kawai, and P. Hänggi, in Statistical Mechanics of Biocomplexity, edited by D. Reguera, J. M. G. Vilar, and J. M. Rubi (Springer-Verlag, Berlin, 1999), pp. 93-111; P. Reimann, Phys. Rep. (to be published), e-print cond-mat/0010237.

[4] R. King, Integrated Electronic Circuits and Systems (Van Nostrand Reinhold, Berkshire, 1983).

[5] P. Reimann, R. Kawai, C. Van den Broeck, and P. Hänggi, Europhys. Lett. 45, 545 (1999); J. Buceta, J. M. R. Parrondo,
C. Van den Broeck, and J. de la Rubia, Phys. Rev. E 61, 6287 (2000); C. Van den Broeck, I. Bena, P. Reimann, and J. Lehmann, Ann. Phys. 9, 713 (2000).

[6] B. Cleuren and C. Van den Broeck, Europhys. Lett. 54, 1 (2001).

[7] D. J. Griffiths, Introduction to Electrodynamics (Prentice Hall, Upper Saddle River, 1989).

[8] J. M. R. Parrondo, G. P. Harmer, and D. Abbott, Phys. Rev. Lett. 85, 5226 (2000).

[9] C. Kettner, P. Reimann, P. Hänggi, and F. Müller, Phys. Rev. E 61, 312 (2000).

[10] J. E. Cohen, Am. Sci. 76, 577 (1988).

[11] T. Alarcon, A. Perez-Madrid, and J. M. Rubi, Phys. Rev. Lett. 85, 3995 (2000).

[12] R. Eichhorn, P. Reimann, and P. Hänggi (unpublished).

[13] N. W. Ashcroft and N. D. Mermin, Solid State Physics (Holt, Rinehart and Winston, New York, 1976). 